\title{
Projeto cuidadores em saúde: um relato de experiência
}

\author{
Health caregivers project: an experience report \\ Proyecto de cuidadores de salud: un informe de experiencia
}

Recebido: 01/11/2021 | Revisado: 09/11/2021 | Aceito: 09/11/2021 | Publicado: 15/11/2021

\author{
Sebastião Rocha \\ ORCID: https://orcid.org/0000-0001-8730-6106 \\ Centro Popular de Cultura e Desenvolvimento, Brasil \\ E-mail: tiaorocha@cpcd.org.br \\ Vânia Lúcia Coutinho \\ ORCID: https://orcid.org/0000-0002-7622-5279 \\ Centro Popular de Cultura e Desenvolvimento, Brasil \\ E-mail:vania@cpcd.org.br \\ Claudia Barbastefano Monteiro \\ ORCID: https://orcid.org/0000-0002-3150-7424 \\ Universidade Federal do Rio de Janeiro, Brasil \\ E-mail:claudia.ipub@gmail.com \\ Cristina Maria Douat Loyola \\ ORCID: https://orcid.org/0000-0003-2824-6531 \\ Universidade CEUMA, Brasil \\ E-mail: crisloyola@hotmail.com
}

\begin{abstract}
Resumo
Objetivo: Este estudo tem como objetivo relatar a experiência do desenvolvimento do Projeto Cuidadores em Saúde em comunidades quilombolas, na formação de cuidadoras leigas no Projeto Cuidadores em Saúde, no estado do Maranhão. Metodologia: Trata-se de um relato de experiência acerca do desenvolvimento e avaliação do projeto para a formação de cuidadores leigos. Resultado: os casos de hipertensão e diabetes foram detectados e acompanhados na comunidade pelos cuidadores, semanalmente, e encaminhados à Estratégia de Saúde da Família para intervenção medicamentosa. Conclusão: Através desta experiencia constatamos que é possível que formar cuidadores leigos com esta carga horária e conteúdos desenvolvidos, para manter em cuidado e monitoramento comunidades quilombolas, quanto à hipertensão, ao diabetes melitos e à síndrome metabólica.
\end{abstract}

Palavras-chave: Cuidadores; Sistema Único de Saúde; Comunidades vulneráveis.

\section{Abstract}

Objective: This study aims to report the experience of the development of the Health Caregivers Project in quilombola communities, in the training of lay caregivers in the Health Caregivers Project, in the state of Maranhão. Methodology: This is an experience report about the development and evaluation of the project for the formation of lay caregivers. Result: cases of hypertension and diabetes were detected and monitored in the community by caregivers, weekly, and referred to the Family Health Strategy for drug intervention. Conclusion: Through this experience, we found that it is possible to train lay caregivers with this workload and developed content, to keep quilombola communities in care and monitoring, regarding hypertension, diabetes mellitus and metabolic syndrome.

Keywords: Caregivers; Unified Health System; Vulnerable groups.

\section{Resumen}

Objetivo: Este estudio tiene como objetivo reportar la experiencia del desarrollo del Proyecto de Cuidadores de Salud en comunidades quilombolas, en la formación de cuidadores laicos en el Proyecto de Cuidadores de Salud, en el estado de Maranhão. Metodología: Se trata de un relato de experiencia sobre el desarrollo y evaluación del proyecto para la formación de cuidadores laicos. Resultado: los casos de hipertensión y diabetes fueron detectados y monitoreados en la comunidad por los cuidadores, semanalmente, y remitidos a la Estrategia de Salud de la Familia para la intervención farmacológica. Conclusión: A través de esta experiencia, encontramos que es posible capacitar a los cuidadores legos con esta carga de trabajo y contenido desarrollado, para mantener a las comunidades quilombolas en el cuidado y seguimiento de la hipertensión, la diabetes mellitus y el síndrome metabólico.

Palabras clave: Cuidadores; Sistema Único de Salud; Comunidades vulnerables. 


\section{Introdução}

O Projeto Cuidadores em Saúde iniciou-se em 2016 no Maranhão nas comunidades quilombolas de Jaibara dos Nogueiras, Oiteiro dos Nogueiras e Pedrinhas, através da parceria firmada entre o Centro Popular de Cultura e Desenvolvimento (CPCD) e a Fundação Vale. Com a parceria renovada em 2017, foi possível a continuidade do projeto naquelas comunidades localizadas ao longo da ferrovia São Luís - Carajás, e ampliada em 2018 para a comunidade de Canta Galo.

O Centro Popular de Cultura e Desenvolvimento é uma organização não governamental sem fins lucrativos, criada em 1984, em Belo Horizonte. Age nas áreas de Educação Popular de Qualidade e Desenvolvimento Comunitário Sustentável, tendo a cultura como matéria-prima e instrumento de trabalho, pedagógico e institucional (Parreira, 2014).

Pesquisas demonstraram uma preocupação com a saúde dos quilombolas e suas suscetibilidades às condições de saúde, como o difícil acesso aos serviços, predominância de doenças, ganho de peso e uma recepção pontual e curativista são algumas das condições que ocorrem nesse grupo populacional (Cardoso, De Melo, \& Freitas, 2018).

O objetivo do Projeto Cuidadores em Saúde em comunidades quilombolas foi formar cuidadores em saúde leigos trazendo uma experiencia inovadora para o Sistema Único de Saúde (SUS) e reafirmando a o foco na promoção da saúde antes da doença característico da Atenção Primária em Saúde, Assim, o projeto promove a saúde naqueles territórios distantes de centros urbanos, aliando-se e reforçando o trabalho dos agentes comunitários de saúde da ESF, porém executando um trabalho dentro das comunidades a partir de transformação social e da união de saberes do campo da enfermagem, da nutrição e da educação física. O foco da atuação dos cuidadores objetiva a educação em saúde e o desenvolvimento comunitário.

É importante ressaltar a gravidade da prevalência dos quadros de diabetes mellitus e de hipertensão nestas comunidades. O diabetes mellitus (DM) e a hipertensão arterial sistêmica (HAS) são doenças crônicas complexas, um grande problema dos tempos atuais e de saúde pública, pelas elevadas prevalência e morbimortalidade a elas associadas. A incidência e a prevalência mundial de DM e do HAS estão associadas a vários fatores, tais como os hábitos alimentares e o uso de sal em grande quantidade, estilo de vida sedentário, excesso de peso, e envelhecimento da população (Garcia et al., 2020).

Em 2017, a Federação Internacional de Diabetes (International Diabetes Federation, IDF) avaliou que 8,8\% da população do mundo com 20 a 79 anos de idade (424,9 milhões de pessoas) vivia com diabetes. O crescimento do diabetes pode ser atribuído à pouca atuação dos sistemas de saúde, ao baixo índice de informação sobre diabetes entre a população geral e ao início insidioso dos sintomas ou progressão do diabetes tipo 2 que pode levar em torno de 10 anos para apresentar sintomas mais evidentes e já apresentando danos e agravos Avalia-se que cerca de 50\% dos casos de diabetes em adultos não sejam diagnosticados e que $84,3 \%$ de todos os casos de diabetes não diagnosticados estejam em países em desenvolvimento (Forti et al., 2019).

Em 2015, foi previsto que a HAS alcançaria 1,13 bilhão de pessoas em todo o mundo. A prevalência cresce gradativamente com a idade, alcançando $60 \%$ da população maior de 60 anos de idade. A hipertensão arterial sistêmica HAS, é um fator de risco independente, linear e contínuo para acidente vascular cerebral AVC, para doenças do coração e para insuficiência renal com necessidade de hemodiálise. O AVC é a principal causa de morte em todas as regiões do Brasil. Os fatores de risco para HAS são: idade, sexo e etnia (maior em afrodescendentes), fatores socioeconômicos uso de sal, álcool e sedentarismo (Garcia et al., 2020; Santos, Prado, Oliveira, \& Almeida-Santos, 2019).

As comunidades quilombolas são grupos, com história própria, semelhanças territoriais específicas e com orgulho da ancestralidade negra junto à resistência da opressão histórica. O grupo quilombola merece destaque por ser um grupo vulnerável socialmente, resultante de um processo histórico de desapropriação de cultura e direitos, sobre quem o efeito refletiu-se nos indicadores de saúde da população (Matos \& Eugenio, 2019). 
A alta taxa de HAS nos quilombolas foi apontada em estudos realizados nos estados da Bahia e do Maranhão, reforçando a necessidade de acesso aos serviços de saúde para prevenção, diagnóstico e manejo adequado da doença. Nas pessoas maiores de 18 anos a HAS esteve associada ao sexo feminino, idade, menor escolaridade, maior renda, uso de medicamentos nos últimos 15 dias, obesidade e diabetes mellitus (Pereira \& Mussi, 2020).

Diante do exposto, este estudo teve por objetivo descrever a experiência do desenvolvimento do projeto para a formação de cuidadores leigos do Projeto Cuidadores em Saúde em comunidades quilombolas, competentes para nomear, acompanhar e cuidar de pessoas com hipertensão e diabetes.

\section{Metodologia}

Trata-se de um estudo de relato de experiência do desenvolvimento do Projeto Cuidadores em Saúde em comunidades quilombolas, na formação de cuidadores em saúde leigos, realizado nas comunidades quilombolas de Jaibara dos Nogueiras, Oitero dos Nogueiras, Pedrinhas e Canta Galo, no Maranhão. O projeto foi pautado na pesquisa-ação participante (PAP) que buscou o resultado prático de problemas, destacando o envolvimento direto e a cooperação dos indivíduos que fizeram parte da comunidade pesquisada (Plomp, Nieveen, Nonato, \& Matta, 2018).

O referencial teórico utilizado teve como base os conceitos de Paulo Freire em que a ação-reflexão-ação, apontam a unidade dialética da práxis, entendendo que esta seja o fazer e o saber reflexivo da ação. O saber que sustenta criticamente o fazer, impacta novamente o saber e, assim, ambos se transformam sucessivamente (Streck, Redim, \& Zitkoski, 2010). A prática docente crítica, envolve o movimento dinâmico, dialético, entre o saber fazer e o pensar sobre o fazer (Freire, 2003).

O sistema de aprendizagem, não deveria ser desenvolvido de cima para baixo, como uma doação ou imposição, mas de dentro para fora, pelo próprio educando, apenas com a colaboração do educador (Freire, 1969). Em uma conexão educativa com um grupo popular, os educandos recomendam os conteúdos propostos que nascem como problemas, encaminhados por uma metodologia. $\mathrm{O}$ educador aproxima-se a partir do problema, interagindo e importando-se com o quadro social e político do respectivo grupo, consolida a interação entre os saberes (Freire \& Nogueira, 1993).

A análise dos dados foi realizada por estatística descritiva utilizada para organizar, resumir e descrever os aspectos importantes de um conjunto de características observadas ou comparar tais características entre dois ou mais conjuntos. As ferramentas descritivas são os muitos tipos de gráficos e tabelas e também medidas de síntese como porcentagens, índices e médias (Mancuso et al., 2018; Reis \& Reis, 2002).

\section{Resultados e Discussão}

A primeira etapa do projeto consistiu na formação de 20 educadores leigos em cada comunidade quilombola, onde foram selecionados os 05 melhores a partir de avaliação teórica prática de desempenho. Estes 05 mais 03 educadores realizaram um mutirão em cada quilombo de modo a detectar e nomear os moradores portadores de hipertensão e diabetes que passaram a ser objeto de cuidado em saúde e encaminhamento à Unidade Básica de Saúde / UBS sempre que os índices glicêmicos e de PA ultrapassaram a normalidade (120/90mmHg e $140 \mathrm{mcg} / \mathrm{ml}$ pós prandial)

\section{Resultados referentes ao controle de pressão arterial e glicemia capilar}

Participaram dessa intervenção 76 quilombolas. A idade dos participantes variou entre 16 e 104 anos, a média de idade foi 60 (+- 15,83\%). Nesse grupo 44 eram mulheres e 32 eram homens.

O Quadro 1 mostra a variação da média da pressão arterial entre os meses de julho de 2017 e janeiro de 2018. Observa-se uma tendencia de redução da pressão arterial sistólica (PAS) e manutenção da pressão arterial diastólica (PAD), porém a análise desses dados não apresentou diferença estatística significativa. 
Quadro 1: Tendência da PA entre quilombolas de julho de 2017 a janeiro de 2018.

\begin{tabular}{|c|c|c|c|c|c|c|c|}
\hline PAS & 155,61 & 156,02 & 154,45 & 150,89 & 146,17 & 140 & 131,39 \\
\hline PAD & 72,20 & 73,61 & 72,44 & 70,78 & 72,87 & 69,82 & 66,42 \\
\hline Mês & Jul & Ago & Set & Out & Nov & Dez & Jan \\
\hline
\end{tabular}

Fonte: Autores.

Para melhor visualização o Gráfico 1 mostra a diminuição gradativa da pressão arterial, entre julho/17 e janeiro/18.

Gráfico 1.

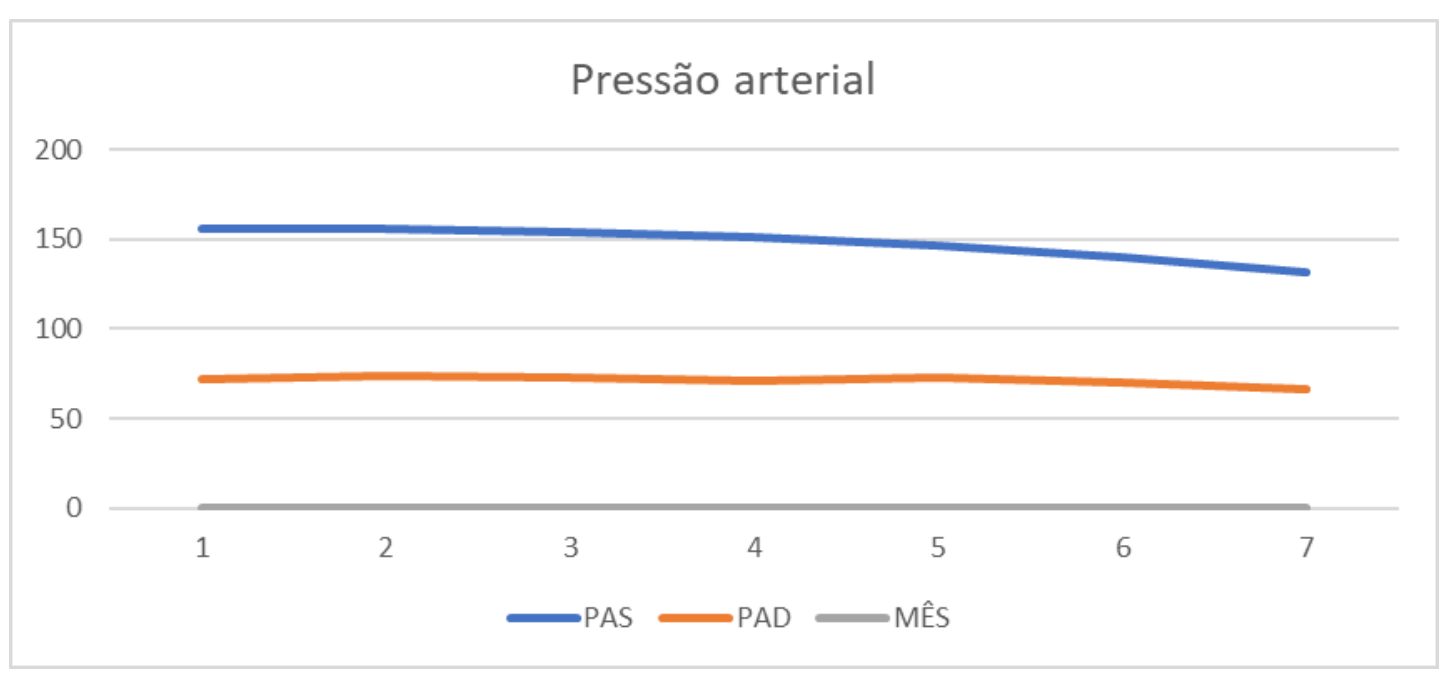

Fonte: Autores.

Dos 76 quilombolas avaliados, 38 apresentavam taxas de glicose capilar alteradas. Nestes, a idade variou entre 29 e 104 anos, a média de idade foi de 60 anos. Quanto ao sexo 24 (63,16 \%) eram mulheres e 14 (36,84\%) eram homens. Na Quadro 2 vislumbra-se sete aferições referentes a taxas de glicose. Observa-se que ocorreu um decréscimo da taxa de glicose, contudo no mês de novembro esta taxa sofreu elevação ${ }^{1}$.

Quadro 2. Tendência da taxa de glicose entre quilombolas de julho de 2017 a janeiro de 2018.

\begin{tabular}{|c|c|c|c|c|c|c|c|}
\hline Glicemia & 167,49 & 160,52 & 154,39 & 151,25 & 154,43 & 138,81 & 135,08 \\
\hline Mês & Jul & Ago & Set & Out & Nov & Dez & Jan \\
\hline
\end{tabular}

Fonte: Autores.

Para melhor visualização o Gráfico 2 mostra a diminuição gradativa da glicemia, entre julho/17 e janeiro/18, com um significativo aumento em novembro.

\footnotetext{
${ }^{1}$ Levamos esta situação para roda de conversa nos quilombos, sobre como explicar aumento de glicemia em todos os quilombos no mês de novembro. A conclusão dos moradores foi a época da manga em fartura, onde as pessoas sentam-se sob a mangueira e come me média 05 mangas, produzindo hiperglicemia.
} 


\section{Gráfico 2.}

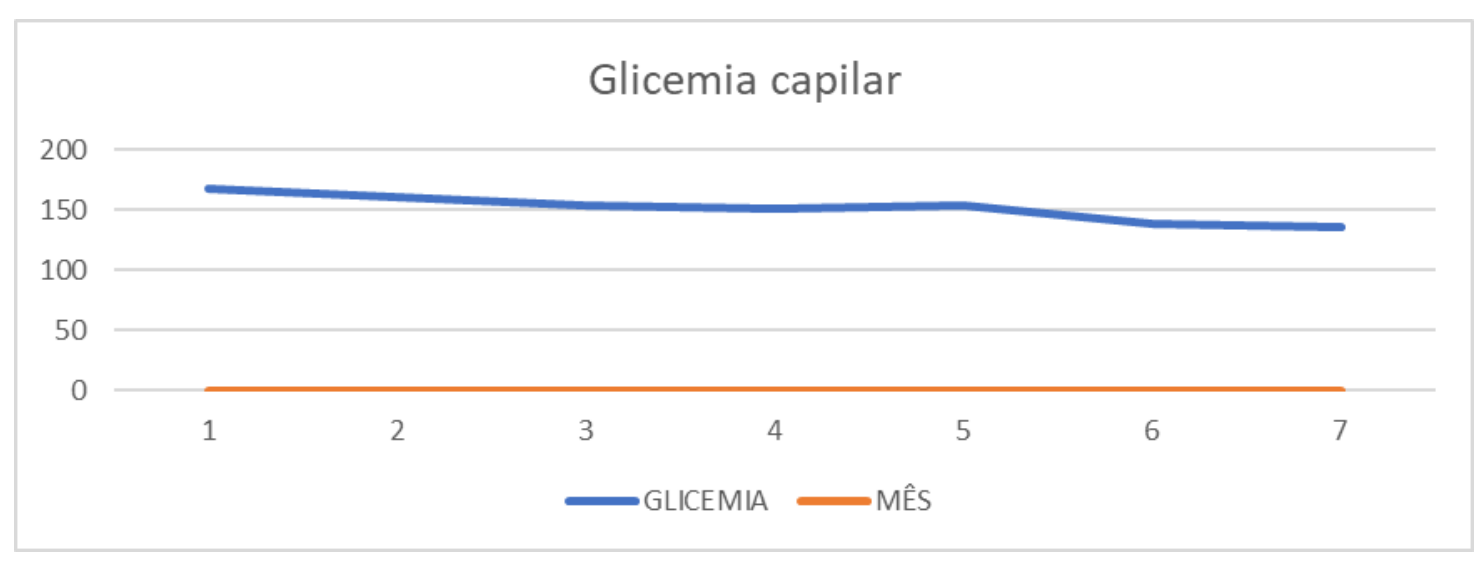

Fonte: Autores.

\section{Resultados referentes a circunferência abdominal}

Participaram dessa intervenção 64 quilombolas, sendo 14 do Jaibara dos Nogueiras, 22 de Pedrinhas e 28 de Oiteiro das Nogueiras. Na Tabela 3 visualizam-se os dados referentes à idade, circunferência abdominal, peso e altura nos 3 quilombos, nos meses de dezembro de 2017, janeiro e fevereiro de 2018. Em Jaibara dos Nogueiras 7,14\% (1) era homem e 13 (92,86\%) eram mulheres, idade média foi $47(18)$ anos, no quilombo de Pedrinhas a média de idade foi de 30 (9,87\%) anos, com 1 pessoa do sexo masculino (4,55\%) e $21(95,45 \%)$ do sexo feminino. O quilombo de Oitero das Nogueiras a média de idade foi de $40(17,47)$ anos, sendo 1 sexo masculino $(3,57 \%)$ e 27 sexos feminino $(96,43 \%)$.

Tabela 3.

\begin{tabular}{|c|c|c|c|c|c|c|}
\hline \multirow{2}{*}{ Variáveis } & \multicolumn{2}{|c|}{ Quilombo 1} & \multicolumn{2}{|c|}{ Quilombo 2} & \multicolumn{2}{|c|}{ Quilombo 3} \\
\hline & & Média & & Média & & Média \\
\hline Idade & 14 & 47 & 22 & 30 & 28 & 40 \\
\hline $\begin{array}{c}\text { Circunferência abdominal } \\
\text { (Dez/17) }\end{array}$ & 14 & 86,83 & 22 & 82,95 & 28 & 83,07 \\
\hline $\begin{array}{l}\text { Circunferência abdominal } \\
\text { (Jan/18) }\end{array}$ & 14 & 85,05 & 22 & 81,80 & 28 & 82,41 \\
\hline $\begin{array}{l}\text { Circunferência abdominal } \\
(\mathrm{Fev} / 18)\end{array}$ & 14 & 81,02 & 22 & 79,90 & 28 & 80,03 \\
\hline Peso (Dez/17) & 13 & 59,13 & 22 & 61,08 & 28 & 61,07 \\
\hline Peso (Jan/18) & 13 & 58,50 & 22 & 59,69 & 28 & 60,33 \\
\hline Peso (Fev/18) & 13 & 54,68 & 22 & 58,22 & 28 & 59,55 \\
\hline Altura & 14 & 1,54 & 22 & 1,54 & 28 & 1,56 \\
\hline
\end{tabular}

Fonte: Autores.

No Gráfico 3 mostramos os valores referentes médios à circunferência abdominal nos três quilombos. Verificou-se uma redução da circunferência abdominal em todos os quilombos entre a primeira e terceira medida 


\section{Gráfico 3.}

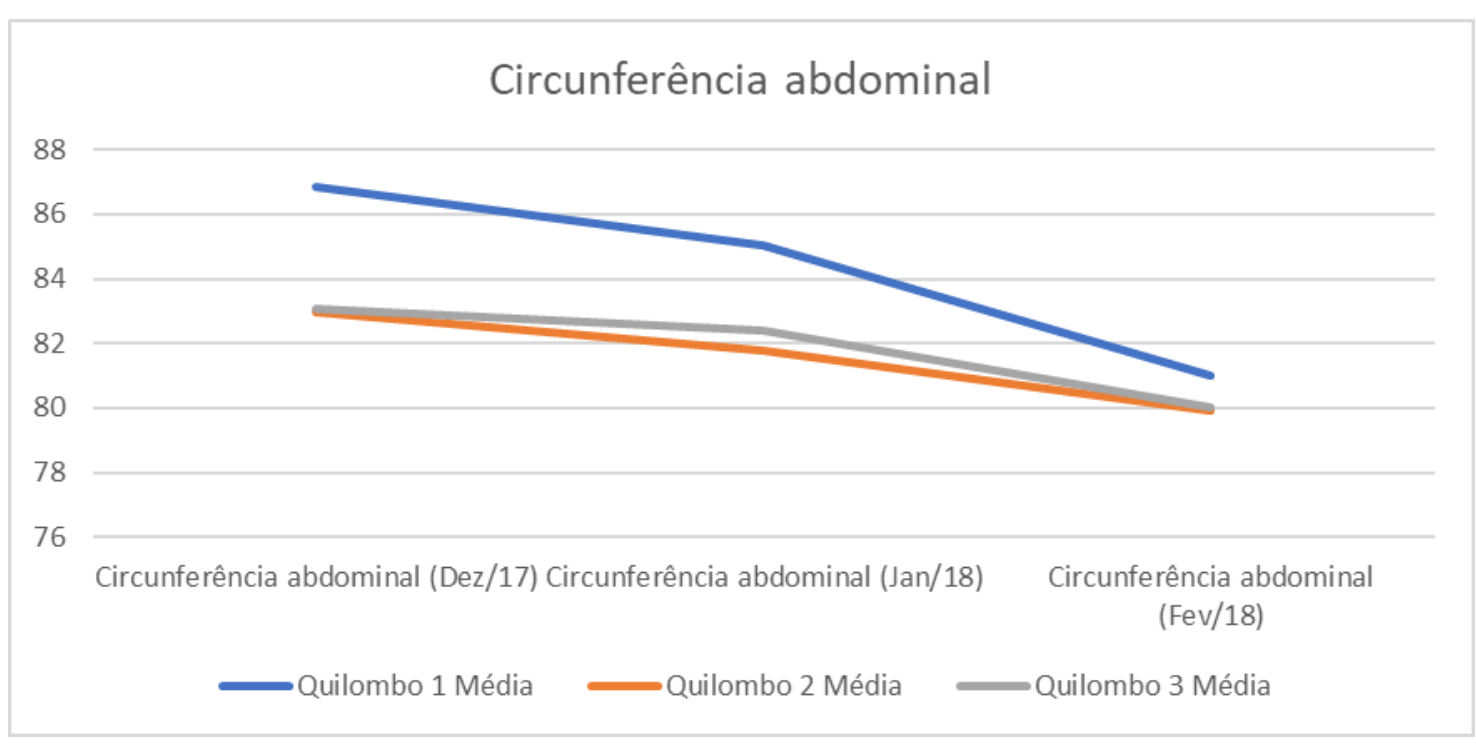

Fonte: Autores.

O Gráfico 4 mostram os valores referentes a média de peso nos três quilombos. Verificou-se uma redução desta variável em todos os quilombos entre as primeiras e terceiras medidas.

Gráfico 4.

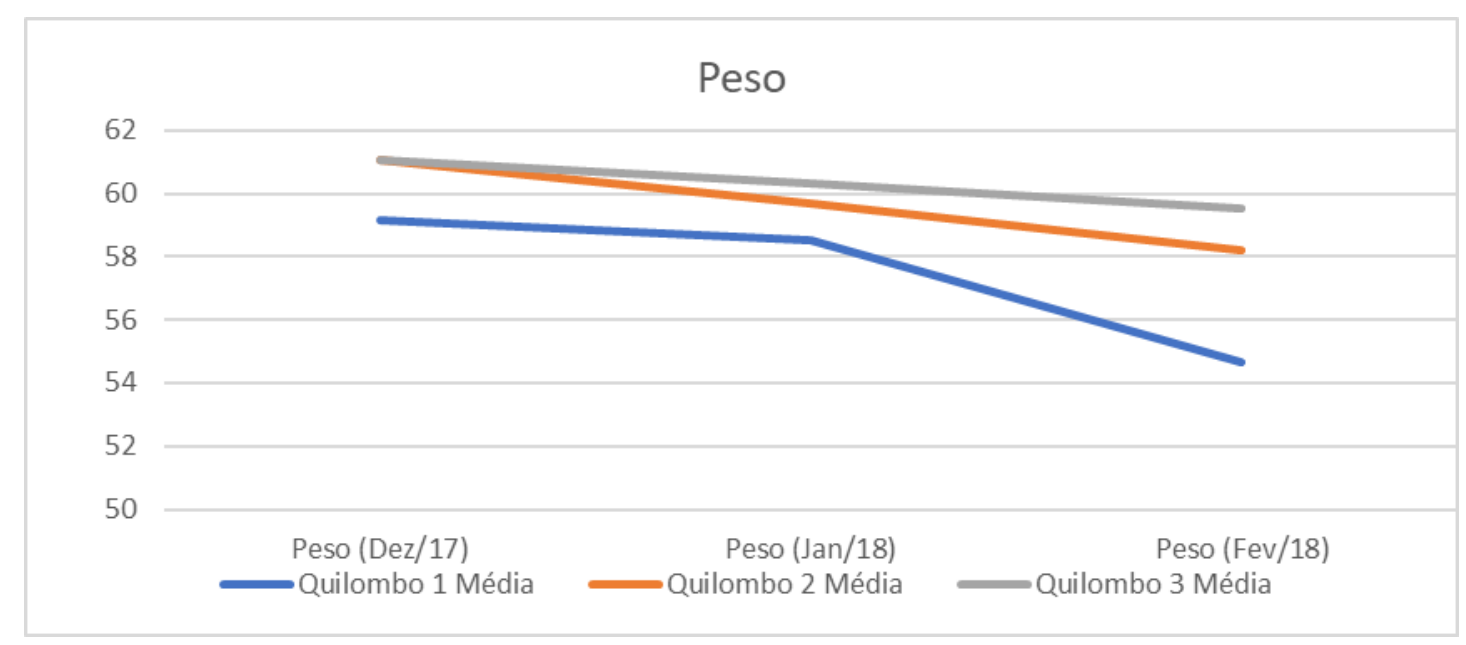

Fonte: Autores.

Os grupos de controle com medição de peso, altura e circunferência abdominal para cálculo do Índice de Massa Corpórea (IMC), foram monitorados através de uma ficha com anotações. Isso possibilitou a visualização concreta das situações individuais e a análise. Depois de cada sessão, as cuidadoras em saúde conversavam com participantes e informavam se estavam dentro da meta prevista, pois cada situação demanda um cuidado específico. O peso não quer dizer, necessariamente, que tenha engordado ou emagrecido. Com os exercícios físicos foi possível ganhar massa muscular, mas o foco estava na análise da diminuição da circunferência abdominal.

O trabalho de campo era iniciado por uma educadora do Centro Popular de Cultura e Desenvolvimento/CPD, que em reunião com os moradores e através de oficinas de formação de agentes comunitários de transformação social de 12 horas, 
selecionava os candidatos a cuidadores leigos. Os professores procuraram a problematização nas oficinas pedagógicas, além da mediação docente, com o objetivo de alcançar superação do senso comum (Fornazari \& Obara, 2017).

O conteúdo clínico sobre verificação da pressão arterial e da glicemia capilar foi desenvolvido em oficinas de 03 dias (24 horas), com ensino teórico prático em roda e com avaliação devolução da prática de uso do aparelho digital de verificação da pressão arterial e do aparelho também digital de mensuração da glicemia pós prandial. Utilizando metodologia assemelhada ensinamos verificação de peso e altura e circunferência abdominal e o cálculo do Índice de Massa Corporal / IMC.

Os 05 alunos com melhor desempenho na parte na oficina eram selecionados como cuidadores leigos nos quilombos em contato permanente com a educadora do CPCD que morava no município de Santa Rita, próximo aos quilombos.

Os aparelhos e matéria necessário foi adquirido através de verba específica da Fundação Vale, parceira do projeto.

As oficinas aconteciam dentro dos quilombos, de um modo geral na Igreja que existe em todos os quilombos. Todos os grupos eram controlados semanalmente pela presença dos cuidadores leigos nos quilombos e pela mensuração das taxas, que aconteciam 02 por semana na Igreja de cada quilombo.

As oficinas desenvolvidas pela nutricionista, em cada quilombo, também contavam com 03 dias ( 24 horas), de atividades teórico-práticas através de atividades lúdicas que se iniciavam com uma "gincana de alimentos" para descobrir e reunir todos os alimentos existentes no quilombo, animais e plantas existentes na comunidade. O objetivo destas oficinas era o de acrescentar fibras à alimentação, e salientar a importância de termos sempre um prato colorido com 05 cores diferentes.

As oficinas desenvolvidas pelo educador físico também obedeciam à mesma carga horária. As atividades tinham o objetivo de criar um ambiente propício e prazeroso para o exercício físico dentro do quilombo. Desenvolvemos um exercício de caminhada para exercício aeróbico ao ar livre pela manhã antes de $09 \mathrm{~h}$, com aquecimento, e mais três conjuntos diferentes de sequência de exercícios ou de dança, de $30 \mathrm{mim}$, envolvendo braços, pernas e tronco, de modo a produzir movimentação corporal e ambiente lúdico. É sabido que a prática de atividades físicas ao ar livre evita o sedentarismo e outros agravos à saúde, considerados sérios problemas para a saúde física e mental (Barancelli \& Pawlowytsch, 2016; Souza et al., 2017).

As oficinas de nutrição, as caminhadas e os exercícios físicos, as bancadas semanais de cuidados e os mutirões de saúde nos quilombos trabalhados, tiveram sempre as salas lotadas o que atestava o sucesso do projeto. As caminhadas eram as mais utilizadas nas comunidades, semanalmente e em grupos de hipertensos e diabéticos. E na comunidade de Oiteiro dos Nogueiras foi muito utilizada a dança tambor de crioula e zumba.

As cuidadoras que se apropriavam das atividades e objetivos do projeto, criaram ferramentas e estratégias como mecanismos para faze-los mudar de atitude. Uma experiencia exemplar foram as oficinas de relaxamento que, naturalmente reduziram a pressão e isso foi comprovado através da mensuração da pressão dos participantes que baixou 02 pontos.

O monitoramento foi a metodologia utilizada para avaliar o resultado individual nos quesitos: PA, glicemia capilar, circunferência abdominal, peso, altura, com índice de massa corpórea (IMC). Com essa avaliação o cliente era orientado sobre as mudanças que deveria fazer na dieta e/ou intensificar os exercícios físicos, associado aos medicamentos recomendados pela ESF.

Para casos resistentes, que não apresentavam nenhuma evolução no quadro, as cuidadoras eram zelosas na orientação e elaboravam um cronograma, planejando junto com a pessoa, o seu dia-a-dia e realizando visita domiciliar no caso de faltas aos exercícios. Essa rotina se fez necessária para garantir a eficácia dos grupos.

As dificuldades foram desde o atendimento precário da saúde nas UBS à resistência pessoal que, aos poucos foram superadas com a determinação da equipe de cuidadoras. Elas participavam periodicamente das capacitações de cuidados em nutrição, dietética e educação física. Promoveram ainda oficinas de utilização de chás naturais com plantas medicinais e terapia de relaxamento por iniciativa própria. Essas práticas resultavam em rodas de conversa com as comunidades no sentido de 
discutir e acrescentar alternativas a fim de atingir o objetivo, superando as limitações socioeconômicas que se apresentavam como fator dificultador no cuidado com a saúde.

Discutir sobre a utilização das ervas naturais no tratamento dessas doenças foi imperativo no trabalho, visto que as comunidades detêm esses saberes através de registro oral, por vezes não valorizado como saber legítimo e com acesso muito restrito (Rodrigues et al., 2020).

Essas experiencias e as oficinas dinamizaram os encontros comunitários e empolgavam as participantes, reforçando que essas práticas deveriam fazer parte da rotina familiar. A prática de "escalda pés", por exemplo, envolveu os familiares que aprenderam nas oficinas e assumiam o compromisso de aplica-las em seus doentes. Essa prática auxilia na diminuição de pressão arterial em até 2 pontos, a redução do estresse e um relaxamento ao final da atividade além de manter o vínculo familiar e a estabilização do quadro do paciente (Silva et al., 2021).

O treinamento das cuidadoras foi facilitado pela parceria com assistentes sociais do município de Itapecuru Mirim e pela equipe da saúde da família de Anajatuba que atende à comunidade e pela boa aceitação da própria comunidade no envolvimento das atividades do projeto, dando visibilidade a ele. Com isso houve aumento da auto estima da comunidade, maior inclusão social, e apropriação e ampliação pelas comunidades no acolhimento e proteção de seus doentes.

O desafio do projeto foi manter o controle da pressão arterial e da glicose, pois a falta de medicamento diários UBS (faltava insulina por exemplo) dificultou melhores de resultados, sendo necessário cuidado redobrado com exercícios físicos, suspensão do sal e açúcar na alimentação para a diminuição do impacto das doenças.

\section{Considerações Finais}

A expectativa é que as comunidades consigam continuar com o cuidado em saúde ainda que sem a parceria da Fundação Vale que descontinuou o projeto que atendia em torno de 100 indivíduos portadores de hipertensão, diabetes ou síndrome metabólica ao longo de 03 anos o projeto apresentou indicadores de eficácia visíveis nas comunidades e público envolvidos, melhoram do as condições de saúde e o sentimento comunitário de responsabilidade e de solidariedade entre pares

As oficinas ministradas por vários profissionais como: enfermeira, professor, educador físico e nutricionista visaram a garantir a estabilidade dos níveis de glicemia e de controle de pressão arterial, através da presença permanente dos cuidadores nos quilombos, produzindo também transformação social, empoderamento das populações e participação articulada.

Através desta experiencia constatamos que é possível que formar cuidadores leigos com esta carga horária e conteúdos desenvolvidos, para manter em cuidado e monitoramento comunidades quilombolas, quanto à hipertensão, ao diabetes melitos e à síndrome metabólica.

Futuramente, a ideia é expandir este modelo de formação de cuidadores para outros quilombos e investir em pesquisaação participante em um novo projeto para formação de cuidadores homens para detecção de câncer de pênis e hanseníase que são de grande prevalência (Nogueira, Nogueira, Pacheco, Monteiro, \& Loyola, 2021).

\section{Referências}

Barancelli, L. L. R., \& Pawlowytsch, P. W. da M. (2016). Dança e qualidade de vida: um estudo biopsicossocial. Repertório, 26(1), $273-282$.

Cardoso, C. S., De Melo, L. O., \& Freitas, D. A. (2018). Condições de saúde nas comunidades quilombolas. Revista de Enfermagem UFPE on Line, 12(4), 1037. https://doi.org/10.5205/1981-8963-v12i4a110258p1037-1045-2018

Fornazari, V. B. R., \& Obara, A. T. (2017). O Uso De Oficinas Pedagógicas Como Estratégia De Ensino E Aprendizagem: a Bacia Hidrográfica Como Tema De Estudo. Investigações Em Ensino de Ciências, 22(2), 166. https://doi.org/10.22600/1518-8795.ienci2017v22n2p166

Forti, A. C. e, Pires, A. C., Pittito, B. de A., Gerchman, F., Oliveira, J. E. P. de, Zajdenverg, L., \& Lottenberg, S. A. (2019). Diretrizes Sociedade Brasileira de Diabetes 2019-2020. Clannad. https://doi.org/10.48075/ra.v8i2.26774

Freire, P. (1969). Educação como prática da liberdade. (P. e Terra, Ed.). 
Research, Society and Development, v. 10, n. 15, e09101522639, 2021

(CC BY 4.0) | ISSN 2525-3409 | DOI: http://dx.doi.org/10.33448/rsd-v10i15.22639

Freire, P. (2003). Pedagogia da autonomia: Saberes necessários à prática educativa. (Paz e Terra, Ed.) (25th ed.).

Freire, P., \& Nogueira, A. (1993). Quefazer: teoria e prática em educação popular. (E. Vozes, Ed.) (4th ed.).

Garcia, É. C., Filho, J. A. B., Matos, M. A. B. de, Lopes, P. R. R., Chomatas, E. R. da V., \& Barra, R. P. (2020). Saúde da pessoa com diabetes mellitus e hipertensão arterial sistêmica. (S. B. I. B. A. Einstein, Ed.).

Mancuso, A. C. B., Castro, S. M. de J., Guimarães, L. S. P., Leotti, V. B., Hirakata, V. N., \& Camey, S. A. (2018). Estatística descritiva: perguntas que você sempre quis fazer, mas nunca teve coragem. Clinical \& Biomedical Research, 38(4), 414-418. https://doi.org/10.4322/2357-9730.89242

Matos, W. S. de., \& Eugenio, B. G. (2019). Comunidades quilombolas: elementos conceituais para sua compreensão. PRACS: Revista Eletrônica de Humanidades Do Curso de Ciências Sociais Da UNIFAP, 11(2), 141. https://doi.org/10.18468/pracs.2018v11n2.p141-153

Nogueira, L. R., Nogueira, R. M. da R., Pacheco, M. A. B., Monteiro, C. B., \& Loyola, C. M. D. (2021). Tamanho é documento: narrativas de homens que sofreram amputação por câncer de pênis. Research, Society and Development, 10(13), e398101321454. https://doi.org/10.33448/rsd-v10i13.21454

Parreira, L. C. A. (2014). Interfaces entre a gestão social e a educação: estudo de caso no centro popular de cultura e desenvolvimento - CPCD. PUC-SP.

Pereira, R. das N., \& Mussi, R. F. de F. (2020). Acesso e utilização dos serviços de saúde da população negra quilombola: uma análise bibliográfica. Odeere, 5(10), 280-303. https://doi.org/10.22481/odeere.v5i10.6938

Plomp, T., Nieveen, N., Nonato, E., \& Matta, A. (2018). Pesquisa-Aplicação Em Educação uma introdução. (ABDE, Ed.).

Reis, E. A., \& Reis, I. A. (2002). Análise Descritiva de Dados. Universidade Federal de Minas Gerais, Instituto de Ciencias Exatas, Departamento de Estatística, 1, 36 .

Rodrigues, T. D. A., Barbosa, M. E., Neto, J. L., Guedes, J. C., Carvalho, T. de A. R., \& Carvalho, A. V. de. (2020). A valorização das plantas medicinais como alternativa à saúde : um estudo etnobotânico Valuing medical plants as an alternative to health : an ethnobotanic study Segundo Torres ( 2009 ), a planta é muito mais o núcleo pedagógico da educação popular para a. Revista Ibero-Americana de Ciências Ambientais, 11(1), 411-428. https://doi.org/10.6008/CBPC2179-6858.2020.001.0037

Santos, D. M. S., Prado, B. S., Oliveira, C. C. da C., \& Almeida-Santos, M. A. (2019). Prevalence of systemic arterial hypertension in quilombola communities, state of Sergipe, Brazil. Arquivos Brasileiros de Cardiologia, 113(3), 383-390. https://doi.org/10.5935/abc.20190143

Silva, F. S. da., Persigo, A. L. K., Mielcke, L., Pagno, A., Rodrigues, Cargnin, M. B., \& Piana, M. (2021). Escalda pés como promotor de saúde em trabalhadores da área da saúde. In Congresso Internacional em Saúde (pp. 3-4).

Souza, F. da S., Reis, D. F. dos, Jesus, J. da S., Garcia, T. A., Ozaki, G. A. T., Zanuto, E. A. C., \& Camargo, J. C. S. (2017). Atividade física ao ar livre e a influencia na qualidade de vida. Colloquium Vitae, 9(Especial), 191-201. https://doi.org/10.5747/cv.2017.v09.nesp.000319

Streck, D., Redim, E., \& Zitkoski, J. J. (orgs). (2010). Dicionário Paulo Freire. (Autentica, Ed.) (2nd ed.). 